\title{
Intraoperative microvascular Doppler ultrasonography in cerebral aneurysm surgery
}

\author{
Ruediger Stendel, Terttu Pietilä, Ali Abo Al Hassan, Andreas Schilling, Mario Brock
}

\begin{abstract}
Objectives-Outcome of surgical treatment of cerebral aneurysms may be severely compromised by local cerebral ischaemia or infarction resulting from the inadvertent occlusion of an adjacent vessel by the aneurysm clip, or by incomplete aneurysm closure. It is therefore mandatory to optimise clip placement in situ to reduce the complication rate. The present study was performed to investigate the reliability of intraoperative microvascular Doppler ultrasonography (MDU) in cerebral aneurysm surgery, and to assess the impact of this method on the surgical procedure itself.
\end{abstract}

Methods-Seventy five patients (19 men, 56 women, mean age $\mathbf{5 4 . 8}$ years, range 22-84 years) with 90 saccular cerebral aneurysms were evaluated. Blood flow velocities in the aneurysmal sac and in the adjacent vessels were determined by MDU before and after aneurysm clipping. The findings of MDU were analysed and compared with those of visual inspection of the surgical site and of postoperative angiography. Analysis was also made of the cases in which the clip was repositioned due to MDU findings.

Results-A relevant stenosis of an adjacent vessel induced by clip positioning that had escaped detection by visual inspection was identified by Doppler ultrasonography in 17 out of 90 (18.9\%) aneurysms. In addition, Doppler ultrasound demonstrated a primarily unoccluded aneurysm in 11 out of $90(12.2 \%)$ patients. The aneurysm clip was repositioned on the basis of the MDU findings in 26 out of $90(28.8 \%)$ cases. In middle cerebral artery (MCA) aneurysms, the MDU results were relevant to the surgical procedure in 17 out of $44(38.6 \%)$ cases. Whereas with aneurysms of the anterior cerebral artery significant findings occurred in only five of 32 cases $(15.6 \%$; $p<0.05)$. The clip was repositioned on the basis of the MDU results in 18 out of 50 $(36 \%)$ aneurysms in patients with subarachnoid haemorrhage (SAH) grade I-V compared with only eight out of $40(20 \%)$ aneurysms in patients without SAH $(\mathbf{p}<0.05)$.

Conclusions-MDU should be used routinely in cerebral aneurysm surgery, especially in cases of MCA aneurysms and after SAH. Present data show that a postoperative angiography becomes superflu- ous whenever there is good visualisation of the "working site" and MDU findings are clear.

(F Neurol Neurosurg Psychiatry 2000;68:29-35)

Keywords: microvascular Doppler ultrasonography; cerebral aneurysm; subarachnoid haemorrhage

The outcome of surgical treatment of cerebral aneurysms may be severely impaired by local cerebral ischaemia, or by infarction resulting from the inadvertent occlusion of an adjacent vessel. Incomplete aneurysm occlusion, on the other hand, increases the risk of haemorrhage. It is therefore of the utmost importance that both aneurysm occlusion and inadvertent clipping of neighbouring vessels be avoided during the surgical act.

Doppler ultrasonography was first used for the assessment of cerebral haemodynamics in extracranial vessels. Aaslid et $a l^{1}$ modified this technique for the transcranial investigation of cerebral vessels. Technical progress made it possible to reduce the size of the ultrasound probe by increasing the ultrasound frequency. Further studies of Nornes et $a l^{2}$ and Gilsbach ${ }^{3}$ led to the development of microprobes for direct investigation of small cerebral vessels.

The present study was performed to investigate the reliability and practicability of intraoperative microvascular Doppler ultrasonography (MDU) during cerebral aneurysm surgery, and to assess the influence of this method on the surgical procedure.

\section{Material and methods}

PATIENTS

Seventy five patients (19 men, 56 women, mean age 53.8 years, range $22-84$ years) with saccular cerebral aneurysms treated by clipping from February 1996 until December 1998 were included in this prospective study. A total of 90 aneurysms were clipped by experienced neurosurgeons. Eighty eight aneurysms were clipped directly. In two cases, an additional wrapping was performed as the clip could not be applied without kinking of the parent artery. The patient data are given in table 1 .

METHODS

Patients were operated on using controlled ventilation, propofol and alfentanil, and muscle relaxation. Blood flow velocities in the aneurysmal sac and adjacent vessels were determined before and after clipping of the aneurysm using intraoperative microvascular Doppler ultrasonography (MDU). The Doppler measurements were performed with a Multidop P (DWL GmbH, Würzburg, Germany) and 
Table 1 Patient details

\begin{tabular}{llll}
\hline & Total $n(\%)$ & $M n(\%)$ & $F n(\%)$ \\
\hline No of patients & $75(100)$ & $19(25.3)$ & $56(74.7)$ \\
Age (y) & 54.8 & 53.6 & 56.1 \\
Range & $22-84$ & $22-73$ & $29-84$ \\
Single aneurysm & $64(85.3)$ & $17(22.7)$ & $47(62.7)$ \\
₹2 aneurysms & $11(14.7)$ & $2(2.7)$ & $9(12.0)$ \\
Total number of aneurysms & 90 & 21 & 69 \\
SAH grade (Hunt and Hess $\left.{ }^{20}\right)$ & $30(40.0)$ & $4(5.3)$ & $26(34.7)$ \\
$\quad 0$ & $45(60.0)$ & $15(20.0)$ & $30(40.0)$ \\
I-V & & & \\
\hline
\end{tabular}

$\mathrm{M}=$ male $; \mathrm{F}=$ female $\mathrm{SAH}=$ subarachnoid haemorrhage.

flexible $16 \mathrm{MHz}$ probes with diameters of 1 $\mathrm{mm}$ used in the pulsed wave mode. The Doppler probe was inserted through a suction cannula to ensure stiffness and to allow precise positioning. The probe was applied to all exposed vessels adjacent to the aneurysm as well as to the aneurysmal sac proper with an insonation angle of 30 to 60 degrees.

Most patients (51 out of 75 ; $68 \%$ ) underwent postoperative angiography, which served as the gold standard for assessing complete occlusion of the aneurysm and patency of adjacent vessels. In six out of $75(8 \%)$ cases, the intraoperative findings were inconclusive or a residual neck below the aneurysm was suspected, so that an intraoperative angiography was performed.

Findings from MDU were analysed and compared with the findings of visual inspection and of intraoperative and postoperative angiography. To evaluate the influence of MDU on the surgical procedure, an analysis was made of the cases in which the clip was repositioned due to unexpected MDU results. Statistical analysis was performed using the $\chi^{2}$ test.

DETECTION OF VESSEL STENOSIS

Qualitative analysis comprised evaluation of the wave form, flow spectrum, and of the acoustic signal. Any alterations of the flow spectrum compared with the initial values, such as lack of the "systolic window", shift of the amplitude maximum to lower frequencies, and inverse flow $^{5}$ associated with typical acoustic phenomena were considered pathological.

Quantitative analysis included measurement of systolic, mean and diastolic blood flow velocities at all of the above sites as well as the calculation of pulsatility and resistance indices. The vessel segments directly at the point of a possible stenosis and proximal and distal to it were insonated.

The following features were considered characteristic for vessel stenosis ${ }^{6}$ :

Table 2 Findings of intraoperative microvascular Doppler ultrasonography (MDU) and their influence on the surgical procedure in 75 patients with 90 aneurysms

\begin{tabular}{lcccc}
\hline Aneurysm location & $\begin{array}{l}\text { No of } \\
\text { aneurysms } \\
n(\%)\end{array}$ & $\begin{array}{l}\text { Insufficient } \\
\text { aneurysm closure } \\
n(\%)\end{array}$ & $\begin{array}{l}\text { Stenosis of } \\
\text { adjacent vessel } \\
n(\%)\end{array}$ & $\begin{array}{l}\text { Repositioning of } \\
\text { aneurysm clip } \\
n(\%)\end{array}$ \\
\hline ACA & $32(35.6)$ & $3(9.4)$ & $3(9.4)$ & $5(15.6)^{\star}$ \\
MCA & $44(48.9)$ & $6(13.6)$ & $12(27.3)$ & $17(38.6)^{\star}$ \\
ICA, PCA, PCoA & $10(11.1)$ & $1(10.0)$ & $1(10.0)$ & $2(20.0)$ \\
BA, AICA, SCA & $4(4.4)$ & $1(25.0)$ & $1(25.0)$ & $2(50.0)$ \\
Total & $90(100)$ & $11(12.2)$ & $17(18.9)$ & $26(28.8)$
\end{tabular}

${ }^{\star} \mathrm{p}<0.05$.

$\mathrm{ACA}=$ anterior cerebral artery (including anterior communicating artery); $\mathrm{MCA}=$ middle cerebral artery; $\mathrm{ICA}=$ internal carotid artery; $\mathrm{PCA}=$ posterior cerebral artery; $\mathrm{PCoA}=$ posterior communicating artery; $\mathrm{BA}=$ basilar artery; $\mathrm{AICA}=$ anterior inferior cerebellar artery; $\mathrm{SCA}=$ superior cerebellar artery.
At the point of the possible stenosis

There was an increase in blood flow velocity compared with the initial values. In cases of very high grade stenosis a decrease in blood flow velocity may occur.

\section{Proximal to the stenosis}

There was a decrease in diastolic blood flow velocity and increase in pulsatility index compared with the values before clipping.

\section{Distal to the stenosis}

A decrease in systolic blood flow velocity and decrease in pulsatility index occurred compared with the values before clipping.

A decrease or increase in flow velocity of at least $10 \%$ was considered significant. Detection of any stenosis by qualitative or quantitative criteria led to repositioning of the clip.

\section{Detection of incomplete aneurysm closure}

Any flow within the aneurysm sac was considered indicative of incomplete closure.

\section{Results}

DETECTION OF VESSEL STENOSIS

Qualitative analysis using MDU showed stenosis of adjacent vessels induced by the clip in 17 out of $90(18.9 \%)$ aneurysms.

Preclipping blood flow velocities within the different vessel segments showed variations due to specific anatomical relations (for example, hypoplastic vessel segments), the influence of anaesthetics, and the subarachnoid haemorrhage. So in quantitative analysis, direct comparison of blood flow velocities before and after clipping was shown to be more useful for practical work, and "normal values" cannot be provided. Quantitative changes were associated with qualitative changes at the point of the stenosis in all cases. Distal to a stenosis quantitative changes were found without associated alterations of the Doppler spectrum in five out of $17(29.4 \%)$ cases with stenosis of an adjacent vessel.

A more differentiated analysis with determination of the grade of stenosis ${ }^{8}$ was not found to be useful.

An initial stenosis of an adjacent vessel induced by clip positioning that had escaped detection by visual inspection was identified during surgery by quantitative analysis in 17 out of $90(18.9 \%)$ aneurysms. The problem was solved by clip repositioning in 15 cases, and by repositioning plus wrapping in two. There was a remarkably (but not significant) higher incidence of vessel stenosis in aneurysms of the middle cerebral artery (12 out of $44 ; 27.3 \%$ ) compared with those of the anterior cerebral artery (three out of $32 ; 9.4 \%$ ) and of the internal carotid artery/posterior communicating artery (one out of $10 ; 10 \%$, table 2). The same holds for MDU findings in patients with SAH compared with those without SAH (table 3).

DETECTION OF INCOMPLETE ANEURYSM CLOSURE By contrast with the results of inspection through the surgical microscope, Doppler ultrasound demonstrated primary incomplete 
Table 3 Findings of intraoperative microvascular Doppler ultrasonography (MDU) and their influence on the surgical procedure in 75 patients with 90 aneurysms in relation to $S A H$ grading according to Hunt and Hess ${ }^{20}$

\begin{tabular}{lllcl}
\hline SAH Grade ${ }^{20}$ & $\begin{array}{l}\text { Number of } \\
\text { aneurysms } \\
n(\%)\end{array}$ & $\begin{array}{l}\text { Insufficient } \\
\text { aneurysm closure } \\
n(\%)\end{array}$ & $\begin{array}{l}\text { Stenosis of } \\
\text { adjacent vessel } \\
n(\%)\end{array}$ & $\begin{array}{l}\text { Repositioning of } \\
\text { aneurysm clip } \\
n(\%)\end{array}$ \\
\hline 0 & $40(44.4)$ & $3(7.5)$ & $5(12.5)$ & $8(20.0)^{\star}$ \\
I-V & $50(55.6)$ & $8(16.0)$ & $12(24.0)$ & $18(36.0)^{\star}$ \\
Total & $90(100)$ & $11(12.2)$ & $17(18.9)$ & $26(28.9)$ \\
\hline${ }^{*}<0.05$. & & &
\end{tabular}

$\mathrm{SAH}=$ subarachnoid haemorrhage.

occlusion in 11 out of $90(12.2 \%)$ aneurysms. In nine $(10 \%)$, clip repositioning resulted in complete occlusion of the aneurysm. In two cases $(2.2 \%)$, additional wrapping became necessary as it was not possible to achieve complete clipping and simultaneous complete patency of adjacent vessels (table 2).

INFLUENCE OF MDU RESULTS ON THE SURGICAL PROCEDURE

The aneurysm clip was repositioned based on the MDU findings in 26 out of 90 (28.8\%) aneurysms. In aneurysms of the middle cerebral artery, the MDU results influenced the surgical procedure in 17 out of $44(38.6 \%)$ cases. This difference was statistically significant $(p<0.05)$ compared with aneurysms of the anterior cerebral artery (five out of $32 ; 15.6 \%$, table 2).

The clip was repositioned based on the MDU results in 18 out of 50 (36\%) aneurysms in patients with SAH grade I-V compared with only eight out of $40(20 \%)$ aneurysms in patients without SAH. This difference was also statistically significant $(\mathrm{p}<0.05$, table 3$)$.

Postoperative angiography was performed in 51 out of $75(68.0 \%)$ patients. Findings from MDU were confirmed by postoperative angiography in all. In six out of $75(8 \%)$ patients intraoperative angiography had to be performed. All MDU findings (free patency of adjacent vessels and complete closure of the aneurysmal sac) were also confirmed in these instances. No complications occurred due to the use of MDU. There were no misleading data from MDU.

In 24 out of 75 (32\%) patients, no postoperative angiography could be performed due to the critical condition of the patient (10 out of $75 ; 13.3 \%$ ), refusal by the patient (six out of $75 ; 8 \%)$, or the clear intraoperative site ( 8 out of $75 ; 10.7 \%$ ).

The mean duration of MDU was 5.2 minutes ( 3 to 7.4 minutes). The intraoperative digital subtraction angiography lasted $38 \mathrm{~min}$ utes on average (29 to 49 minutes).

\section{Discussion}

TECHNIQUE OF MDU

The pulsed wave devices used for MDU allow depth focusing on the region of interest, thus avoiding artefacts caused by adjacent vascular structures. When narrowing of an adjacent vessel by the aneurysm clip occurs, there is an immediate increase in systolic and mean flow velocity within the narrowed vessel segment. Furthermore, alterations in Doppler curve spectrum occur. The degree of stenosis can be assessed by qualitative and quantitative criteria. $^{56}$

No change in flow characteristics will occur distal to a stenosis when the stenosis is compensated. On the other hand, noncompensated ("haemodynamically relevant") stenosis will result in a poststenotic reduced blood flow velocity. Systolic blood flow velocity as well as pulsatility is reduced in particular. Proximal to the stenosis a decrease above all in diastolic blood flow velocity will occur accompanied by an increase in pulsatility (figs 1 and 2 A-E). ${ }^{6}$

Alterations in the Doppler spectrum were seen in all cases at the point of stenosis. Distal of a stenosis quantitative changes without associated alterations of the Doppler spectrum were found in five out of $17(29.4 \%)$ cases. This may possibly be caused by the different distances between the stenosis and the point of measurement distal to it. Therefore a purely qualitative study may only be reliable if the point of the possible stenosis is included in the measurements.

Blood flow velocities show variations in different vessels and vessel segments, due to the anaesthesia, and caused by subarachnoid haemorrhage. As there are several criteria for detecting a stenosis which are complementary rather than competitive,,$^{5-7}$ a direct comparison of wave forms, spectrum, and flow velocities before and after clipping-measured at the same points of the vessel-was shown to be more useful for practical work.

The Doppler spectra and flow velocities should be identical before and after clipping. Increase or decrease in flow velocity as well as any change in flow spectrum ${ }^{5}$ has to be regarded as pathological. An exception is the turbulent flow in the vessel segment directly at the attachment of the aneurysm neck, which may disappear after clipping.

A stenosis can certainly be detected by Doppler ultrasonography if the vessel diameter is narrowed more than or about $50 \%$. The stenosis is described as decompensated or "haemodynamically relevant" from about $80 \%$ reduction of the diameter. ${ }^{9}$

Even an additional stenosis of only $30 \%$ caused by vasospasm may lead to decompensation in the case of a pre-existing low grade stenosis of $50 \%$ caused by the aneurysm clip. This situation may result in local cerebral ischaemia. Therefore, any stenosis of a vessel detected by MDU after aneurysm clipping must be avoided.

THE USE OF MDU IN CEREBRAL ANEURYSM SURGERY

Karhunen ${ }^{10}$ reported on the results of postmortem angiography after aneurysm clipping in 63 patients with ruptured aneurysm. Surgical complications of vascular aetiology were encountered in 28 out of $63(44 \%)$ cases. Obstruction of cerebral arteries caused by the aneurysm clip was detected by angiography in 13 out of $63(21 \%)$ cases. Incomplete aneurysm occlusion, on the other hand, may lead to postoperative bleeding or repeat surgery. It is therefore necessary to check the proper clip 

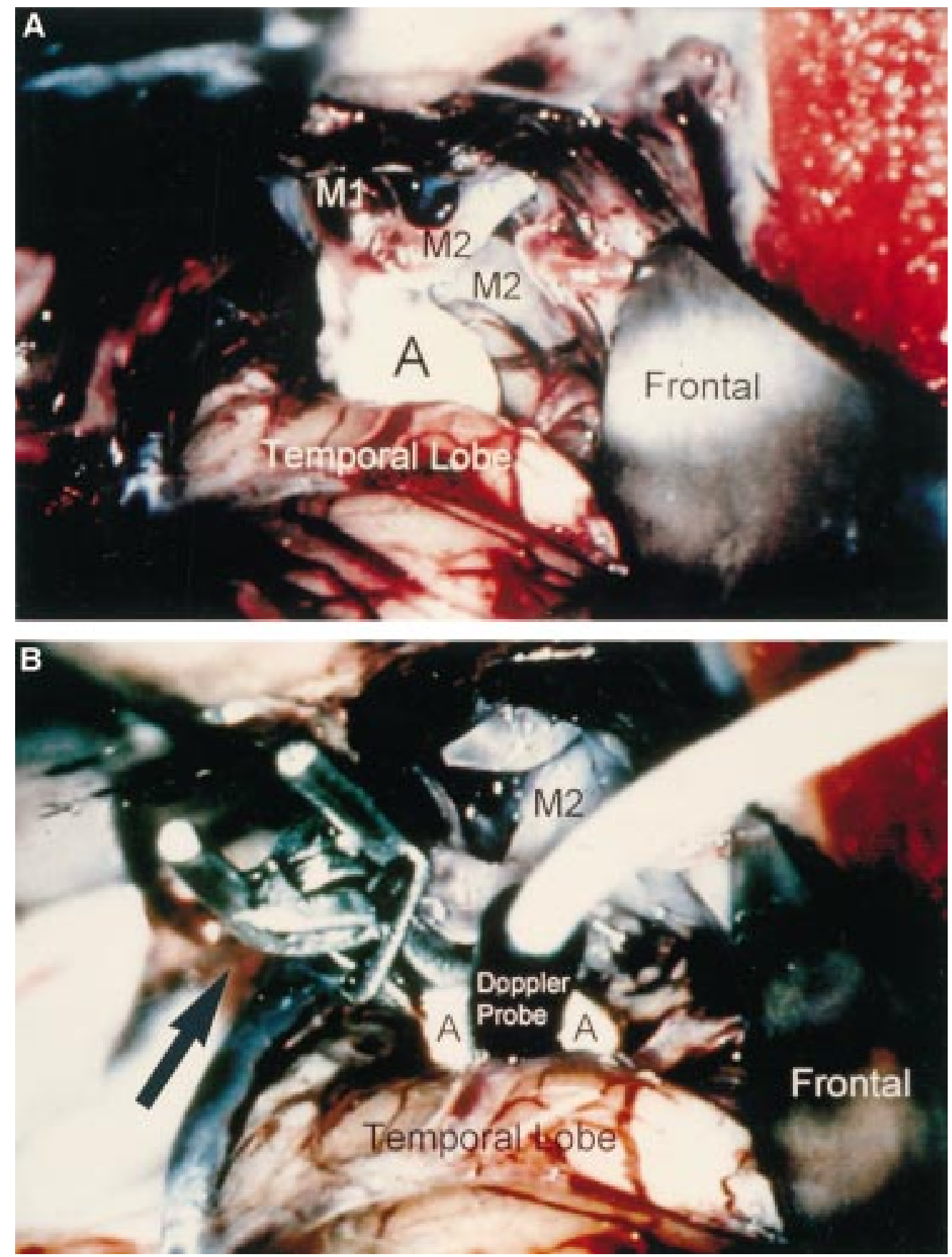

Figure 1 (A) Intraoperative site after dissection of an aneurysm of the middle cerebral artery. The middle cerebral artery trunk (M1) is seen as well as two branches (M2) of the trifurcation. (B) Intraoperative picture showing the clip (arrow) on the neck of the aneurysm and the Doppler probe on the dome of the aneurysm $(A)$.

position and the haemodynamic situation after clipping, preferable during surgery. This has been tried by different techniques.

Visual inspection of the surgical site in cerebral aneurysm surgery has not proved to suffice and cannot replace more extensive studies with complex equipment. ${ }^{11}$ This holds in particular for complex lobulated aneurysms of the anterior communicating artery.

Postoperative digital subtraction angiography is a reliable imaging method, regarded as the gold standard in vascular surgery in general because it shows the cerebral vessels under optimal conditions. MacDonald et $a l^{12}$ studied 78 patients with intracranial aneurysms by postoperative subtraction angiography and identified unexpected stenoses in nine out of $78(11.5 \%)$ patients with subsequent stroke or death in eight of these nine $(10.3 \%)$ patients. The incidence of unexpected residual aneurysms was $4 \%$, and another $4 \%$ of the aneurysms were found not to be clipped at all.

However, postoperative angiography makes a second operation necessary should there be stenoses or incomplete aneurysm closure. Therefore, there is need for a method providing reliable and quick intraoperative control of the quality of the achieved clipping.

Martin $e t a l^{13}$ used intraoperative digital subtraction angiography in 57 patients with cerebral aneurysm and identified five $(8.8 \%)$ improperly placed aneurysm clips. Immediate 

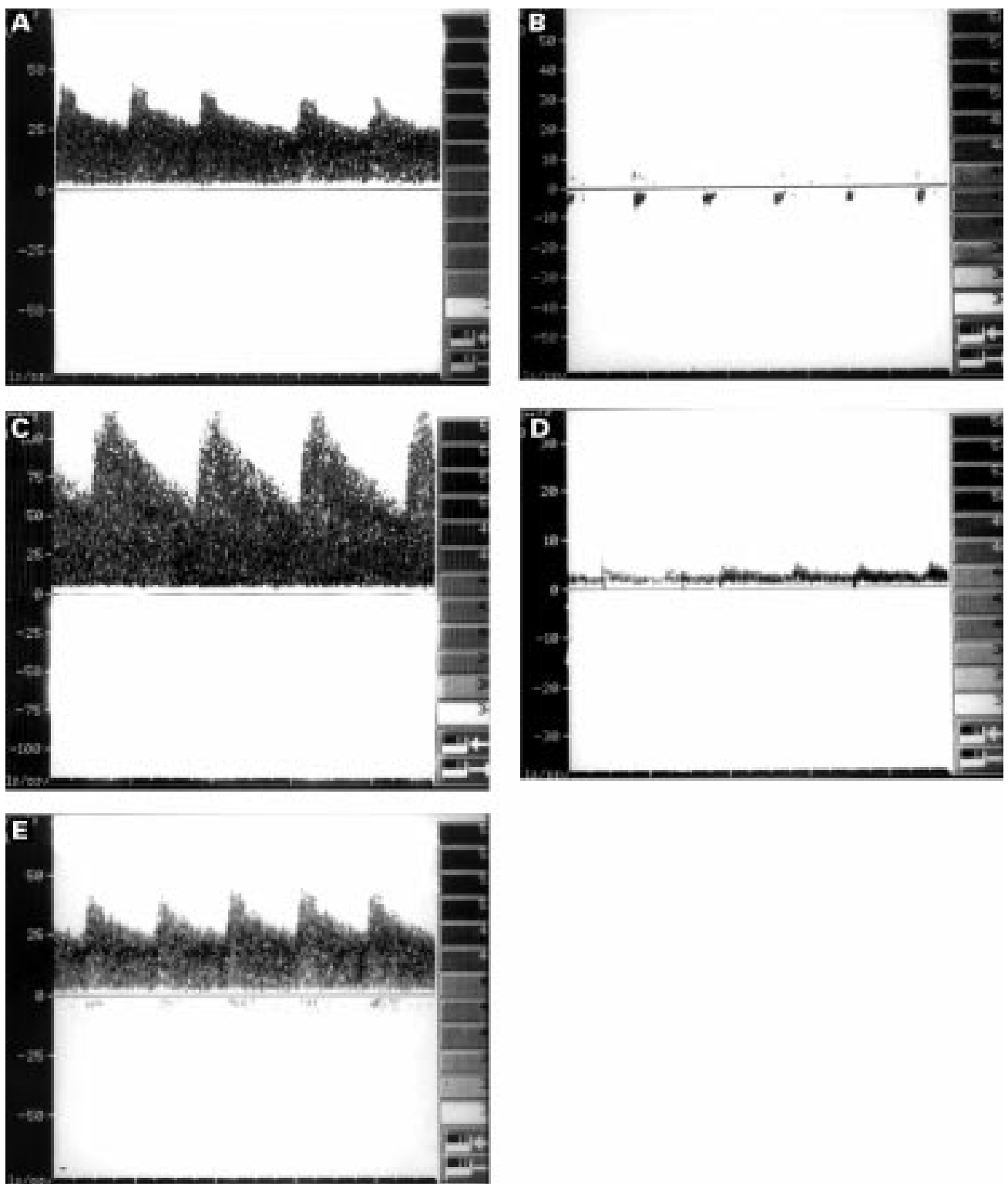

Figure 2 (A) Baseline (preclipping) Doppler curve of an M2 branch of the middle cerebral artery. (B) Persistent systolic intraaneurysmal flow demonstrates incomplete closure of the aneurysmal neck. The aneurysm clip was repositioned. (C) After repositioning of the clip, increase in flow velocity and alteration of the Doppler spectrum indicates stenosis directly at the point where the aneurysm clip is in contact with the M2 branch. The stenosis was not expected by visual inspection. (D) Microvascular Doppler ultrasonography shows a decrease in flow velocity measured on one M2 branch distal to the point where the clip induces stenosis (poststenotic). The Doppler curve is indicating high grade stenosis of the M2 branch. (E) After renewed repositioning of the clip, Doppler ultrasonography shows normal sonographic flow pattern in M2.

revisions were undertaken in all cases. However, the technique is expensive, time consuming, and technically complex. Repeated intraoperative digital subtraction angiography may pose problems. In the series of Martin et al, ${ }^{13}$ postoperative angiography identified two $(3.5 \%)$ patients with residual aneurysm perfusion that had escaped detection by intraoperative digital subtraction angiography.

In addition, the complication rate of angiography is by no means negligible. In a study by Barrow et $a l^{14}$ of 115 patients who underwent intraoperative angiography, complications occurred in two cases $(1.7 \%)$. One of the patients had asymptomatic one sided embolism of A1. The other died from aortic dissection and rupture on the fourth postoperative day. The 105 intraoperative angiographies performed in the series by Martin et $a l^{13}$ were associated with severe complications (permanent neurological deficit) in three cases $(2.9 \%)$.

Another method used intraoperatively during cerebral aneurysm surgery is electrophysiological monitoring. However, this method allows only indirect identification of significant cerebral ischaemia and yields neither information on stenosis of adjacent vessels, nor on the completeness of aneurysm occlusion. In addition, the findings of this procedure often show a wide variation and are affected by anaesthesia. ${ }^{15}$ For aneurysms located in the posterior fossa, Manninen et $a l^{16}$ reported false negative results in $47 \%$ of the monitored patients.

Intraoperative microvascular Doppler ultrasonography (MDU) provides a functional and non-invasive intraoperative examination of single vessel haemodynamics and of the aneurysm proper. It can help to identify the aneurysmal 
sac by typical flow patterns even under complicated anatomic conditions. MDU also allows determination of the flow direction, and detection of vasospasm with a high degree of sensitivity. Furthermore, MDU can be used for assessing vessel patency and direction of flow following trapping bypass and blood flow redirection procedures for complex aneurysms. Microprobes, such as the ones used in the present study allow the examination even of very small vessels such as the perforating arteries. $^{11}$

Bailes et $a l^{11}$ investigated 35 patients who underwent surgery for the treatment of 42 intracranial aneurysms by means of microvascular Doppler ultrasonography. They found an incidence of $31 \%$ for unexpected stenosis of adjacent arteries. However, they did not provide quantitative data on the incidence of insufficient aneurysm closure and its relation to aneurysm location. The findings of Doppler ultrasonography correlated with the findings of angiography in all cases. The authors concluded that microvascular Doppler ultrasonography can replace intraoperative angiography and in many cases postoperative angiography.

The results of the present study support this suggestion. Furthermore, the high incidence of inadequate aneurysm closure detected by MDU in our study adds to the value of the potential benefit for its use in cerebral aneurysm surgery.

In the present study the overall incidence of insufficient aneurysm closure was $12.2 \%$ (11 out of 90 cases), with a non-significant higher incidence after subarachnoid haemorrhage. This may be explained by technical difficulties in surgical preparation caused by the bleeding and subsequent brain swelling. Other reports on the rate of incompletely clipped aneurysms range from $3.8 \%{ }^{17}$ to $8 \%{ }^{18}$ In general, it is necessary to take into account that a minor flow within the aneurysmal sac after clipping may disappear due to thrombosis after a period. In those cases, postoperative angiography would not detect persistent flow within the aneurysm, whereas MDU would.

The incidence of vessel stenosis in the present study amounted to $18.9 \%$ (17 out of 90 cases) and is similar to the results of the postmortem angiographic study by Karhunen ${ }^{10}$ (incidence of vessel stenosis of $21 \%$ ) as described above. Bailes et $a l^{11}$ reported an incidence of clip induced vessel stenosis of $31 \%$ (11 out of 35 patients) in his study with intraoperative microvascular Doppler ultrasonography. This is higher compared with the cited studies and may probably be explained by the few cases investigated.

The present data demonstrate a statistically significant higher influence of the MDU results on the surgical procedure in aneurysms of the middle cerebral artery and after subarachnoid haemorrhage (tables 2 and 3 ). The first may be caused by the special anatomical relations in aneurysms of the middle cerebral artery, the second by the difficult surgical preparation after the bleeding and consecutive brain swelling.
Although MDU is able to verify the complete closure of the aneurysmal sac, it cannot detect a residual neck. However, if this is suspected by visual inspection, MDU can verify or preclude perfusion within it. In the prospective angiographic study of Sindou et al ${ }^{19}$ on 305 clipped aneurysms clipping was considered incomplete in 18 out of 305 (5.9\%) aneurysms. Among these, the group with residual neck amounted to 12 out of 305 (4\%) aneurysms only. A residual neck usually does not lead to revision (if not enlarged after a period), whereas residual aneurysmal sac does in most cases. The incidence of the second group was reported to be only $1.9 \%$ ( 6 out of 305 aneurysms). Taking into account the complication rate of $2.9 \%$ ( 3 out of 105 patients) of intraoperative angiography in the series of Martin et $a l^{13}$ its routine use in cerebral aneurysm surgery may be problematical. However, in cases with suspected residual sac, which can enlarge over time, control by subtraction (or MRI) angiography should be performed.

In rare cases, the precision of the MDU recordings is adversely influenced by an unfavourable high angle of insonation. Removal of the spatula after clipping may lead to brain shifting with changes in the situation of the clip in relation to adjacent vessels. In addition, the aneurysm clip can cause trauma to an adjacent vessel, which can subsequently lead to thrombosis and vessel occlusion. Obviously these "late intraoperative" alterations are not detectable or predictable by previous MDU, and can only be detected by intraoperative or postoperative digital angiography.

The time and cost factors should also be considered. Angiography is expensive (the equipment and contrast media) compared with MDU. It prolongs the duration of surgery by about 38 minutes compared with 4.2 minutes by MDU. Angiography requires additional personnel. Further, it may be associated with costly complications.

MDU, on the other hand, is non-invasive, easy to use, reliable, and inexpensive. It should therefore be used routinely during aneurysm surgery, in particular after SAH. In our experience, MDU may make angiographic control of aneurysm clipping superfluous when there is good visualisation of the operation site and clear MDU findings.

1 Aaslid R, Markwalder TM, Nornes H. Non-invasive ranscranial Doppler ultrasound recording of flow velocity n basal cerebral arteries. F Neurosurg 1982;57:769-74.

2 Nornes H, Grip A, Wikeby P. Intraoperative evaluation of cerebral haemodynamics using directional Doppler technique: 2. Saccular aneurysms. $\mathcal{F}$ Neurosurg 1979;50: 570-7.

3 Gilsbach JM. Mikrovaskulare intraoperative DopplerSonographie. Ultraschall Med 1984;5:246-54.

4 Gilsbach JM, Hassler WE. Intraoperative Doppler and real time sonography in neurosurgery. Neurosurg Rev 1984;7: 199-208.

5 Arbeitskreis Gefäßdiagnostik der DEGUM: Spektrumanalyse von Dopplersignalen hirnversorgender Arterien. Ultraschall 1987;8:112-13.

6 Spencer MP, Reid JM. Quantification of carotid stenosis with continuous-wave Doppler ultrasound. Stroke 1979;10: with cont

7 Ley-Pozo J, Ringelstein EB. Non-invasive detection of occlusive disease of the carotid siphon and middle cerebral artery. Ann Neurol 1990;28:758-65. 
8 Widder B, von Reutern GM, Neuerburg-Heusler D. Morphologische und dopplersonographische Kriterien zur Bestimmung von Stenosierungsgraden an der A carotis interna. Ultraschall 1986;7:70-5.

9 Flanigan PD, Tullis JP, Streeter VL, et al. Multiple subcritical arterial stenosis: effect on poststenotic pressure and flow. Ann Surg 1977;186:663-8.

10 Karhunen PJ. Neurosurgical vascular complications associated with aneurysm clips evaluated by post-mortem angiography. Forensic Sci Int 1991;51:13-22.

11 Bailes JE, Tantuwaya LS, Fukushima T, et al. Intraoperative microvascular Doppler sonography in aneurysm surgery. Neurosurgery 1997;40:965-70.

12 MacDonald RL, Wallace MC, Kestle JRW. Role of angiography following aneurysm surgery. If Neurosurg 1993;79:826-32.

13 Martin NA, Bentson J, Vi×uela F, et al. Intraoperative digital subtraction angiography and the surgical treatment of intracranial aneurysms and vascular malformations. $7 \mathrm{Neu}$ rosurg 1990;73:526-33.
14 Barrow DL, Boyer KL, Joseph GJ. Intraoperative angiography in the management of neurovascular disorders. graphy in the management

15 Friedman WA, Chadwick GM, Verhoeven FJS, et al. Monitoring of somatosensory evoked potentials during surgery for middle cerebral artery aneurysms. Neurosurgery 1991; 29:83-8.

16 Manninen PH, Patterson S, Lam AM, et al. Evoked potential monitoring during posterior fossa aneurysm surgery: a comparison of two modalities. Can F Anaesth 1994;41:92-97.

17 Feuerberg I, Lindquist Ch. Natural history of postoperative aneurysm rests. 7 Neurosurg 1987;66:79-89.

18 Kassell NF. Angiography after aneurysm surgery. 7 Neurosurg 1994;80:953-4.

19 Sindou M, Acevedo JC, Turiman F. Aneurysmal remnants after microsurgical clipping: classification and results from a prospective angiographic study (in a consecutive series of a prospectived acta series of (Wien) 1998;140:1153-9.

20 Hunt WE, Hess RM. Surgical risk as related to time of intervention in the repair of intracranial aneurysms. $\mathcal{F} \mathrm{Neu}$ rosurg 1968;28:14-20.

\section{NEUROLOGICAL STAMP}

\section{Nicholas of Cusa (1401-64)}

Nicholas of Cusa was born in 1401 at Kues (Cusa) which is now in West Germany. He obtained an LLD in 1423 and became a Cardinal in the Roman Catholic Church. Nicholas was a philosopher, scientist, and mathematician. In his most lasting work De Docta Ignorantia (on learned ignorance) published in 1440, he argued against the possibility of definitive knowledge. He made discoveries in astronomy and botany and was said to have been the first to record the use of concave lenses to correct visual defects for the near sighted, thus paving the way for development of modern optometry and the manufacture of spectacles. Benjamin Franklin (1706-90) who appeared earlier in this series ( $F$ Neurol Neurosurg Psychiatry 1993;56:5) invented bifocal lenses.

Nicholas of Cusa was portrayed philatelically in 1984 by Transkei in that country's third set of Heroes of Medicine series (Stanley Gibbons 160, Scott 105).

L F HAAS

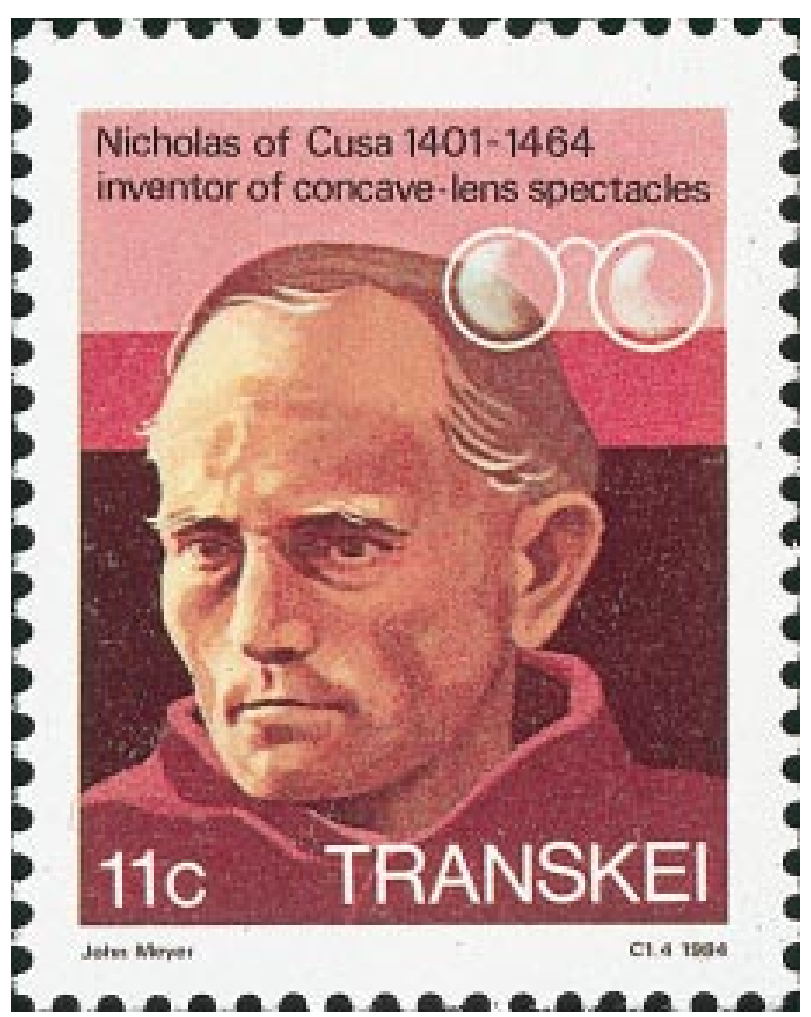

